\title{
INCLUSIVE CULTURAL HERITAGE SITES: ICT AS A TOOL TO SUPPORT THE DESIGN PROCESS AND SHARE KNOWLEDGE
}

\author{
S. Marconcini ${ }^{1}$, V. Pracchi ${ }^{1}$ \\ ${ }^{1}$ Dept. of Architecture, Build environment and Construction Engineering, Politecnico di Milano, Italy \\ sebastiano.marconcini@polimi.it; valeria.pracchi@polimi.it
}

KEY WORDS: Inclusion, Accessibility, Cultural Heritage, ICT, Interactive Map, Management, Information System, Knowledge

\begin{abstract}
:
Within the European strategies for a sustainable development, the role of cultural heritage as an economic, environmental and social driver has become increasingly significant. As an asset in people's lives, it's necessary to assure anyone the opportunity to access it. For this reason, urban transformation policies must guarantee the proper compromise between the requirements of conservation and physical/cognitive accessibility. This entails a clear design complexity, which however cannot justify the lack of intervention, but must propose new governance models for an inclusive design process.

In a broader research framework, the implementation of ICT has turned out to be a solution that can address some issues in enhancing the level of inclusion in cultural heritage sites. Particularly, the conception of an interactive map has seemed the proper perspective of producing a feasible operative tool. The first aim is linked to the necessity of having an information system thank to which everyone, particularly users with special needs, could be able to organize their movements and be aware of the proposed services and fulfilled inclusive strategies. The second target is connected to the management of the projects related to the development of inclusion, therefore it is addressed to municipal administrators and other key actors involved in the governance of cultural heritage.

Finally, the discussion about the main objectives and features of an interactive map wants to highlight the role that ICT can assume within an inclusive design approach, for which is needed a tool able to support the intervention on the physical environment and offer further essential services.
\end{abstract}

\section{INTRODUCTION}

From the 1972 UNESCO World Heritage Convention to the initiatives and events of the European Year of Cultural Heritage (2018), the role of cultural heritage as an economic, environmental and social driver has become increasingly significant. Moreover, the values of $\mathrm{CH}$ have been completely rethought with an emphasis not so much on why should we protect it, as for whom have we got to do it (FARO, 2005). This emphasizes one of the cogent themes of contemporary culture: the notion of cultural good as 'public good' which, for this reason, should be accessible to the widest part of the members of each community. In fact, access to culture is one of the fundamental rights guaranteed by different legal systems, starting with the UN Universal Declaration of Human Rights, which states that "everyone has the right freely to participate in the cultural life of the community, to enjoy the arts and to share in scientific advancement and its benefits" (art. 27). Most of the constitutions of the European Union States, directly or indirectly, refer to the issue of culture accessibility, considered a central issue. The work plan for culture (2015-2018) adopted by the European Council in 2014, also mentions an accessible and inclusive culture as a priority. The importance of achieving a better and more equitable distribution of the possibilities for participation was underlined by a series of Council conclusions (2010) that see culture as a tool, capable of acting also in the fight against poverty, as well as social exclusion.

The intervening evolution is also evident in the changes introduced in 2008 to the Italian Code of Cultural Heritage, which in the definition of "valorization" (art. 6) also includes the interventions aimed "at ensuring the best conditions of use and public enjoyment of the asset itself, also by people with disabilities, in order to promote the development of culture". Starting from this, our intentions is to propose strategies and experiments that can promote profitable forms of relationship between people with special needs and cultural heritage.

\section{TOWARDS A ROLE FOR ICT IN FOSTERING INCLUSIVE POLICIES}

According to these premises, the goal of this paper is to deepen the discussion about the role that ICT could play in fostering public participation in cultural heritage sites. In particular, once understood the needs that inclusive policies must address, the proposal of an operative tool will try to identify how its implementation represents a key resource for an inclusive design approach and which challenges still have to be faced.

\subsection{Inclusive cultural heritage sites: which challenges?}

Given the universal value of cultural heritage, to which everyone should have access, it is important to understand the meaning the term "inclusion" assumes in this work.

On the one hand, the attention is focused on the enhancement of the physical accessibility of the built environment, as a place that can enable or disable people in achieving their functioning and consequently their well-being.

In this regard, a fundamental document in understanding the influence of the built environment on everyone's life is the International Classification of Functioning, Disability and Health (ICF) by the World Health Organization (WHO). In relation to the topic of disability, the ICF represents the latest version of a tool introduced to assess a person's health and wellbeing. The importance of this classification system lies in the acknowledgment of another element, other than the physical conditions, that influences people's functioning and their ability to do personal activities and participate in life situations: 
contextual factors. The latter are also divided into environmental factors, that are the physical and social features of the surroundings in which people live their lives, and personal factors, which involve the particular background of an individual, like the cultural one (WHO, 2001). This connection with the built environment has also been underlined in the United Nations Convention on the Rights of Persons with Disabilities, which defines disability as the result "from the interaction between persons with impairments and attitudinal and environmental barriers that hinder their full and effective participation in society on an equal basis with others" (UN, 2006).

On the other hand, the focus is set on the importance of sociocultural component in defining human well-being and how architecture should foster access to knowledge and public participation, regardless of the cultural background of a person. Culture has appeared as another fundamental need for human beings. In their different forms, socio-cultural factors influence the possibilities of participation in the public realm and human well-being, but the key issue can be identified in the topic of identity. The American psychologist A. Maslow, in developing his "Theory of Human Motivations", among the basic human needs pointed out those of belonging, self-esteem and selfrealization. In our society, all people perceive the need for a stable, solidly based and generally high evaluation of themselves, through their own inclusion within a broader group (Maslow, 1943). A person needs to develop and express, at the same time, his own personal identity and the belonging to some collective system. Since culture is the maximum manifestation of this identity, it is essential that the built environment conveys the latter in its physical features, but especially it has to offers spaces that house services that manifest the culture intangible assets and through which promote public participation and the acquisition of new knowledge; in other words, places that develop identity.

\subsection{Foster inclusion: which role for ICT?}

The previous sections have allowed to better understand how inclusion assumes a specific meaning in relation to cultural heritage sites. In addition to the importance to assure their physical accessibility, it has become clearer which potentialities they have, in turn, in fostering inclusion, especially from a socio-cultural point of view.

Despite the relevance of the topic is widely acknowledged, the current design practice reveals multiple problems in developing inclusion in the architectural project, especially in cultural heritage sites. In order to better comprehend the issues that will be shortly described, it's necessary to highlight how this paper is part of a broader research aimed at develop a fully inclusive view of the project, especially regarding historical contexts. Through the analysis of multiple European case studies and especially an on-site investigation applied to Mantua (a city located in Lombardy region, Northern Italy, entered in the UNESCO World Heritage List in 2008, along with the site of Sabbioneta), it has been possible to identify two main issues that can be addressed by the implementation of ICT in the design process.

First, the management of the applied actions to foster inclusion certainly represents one of the main problems. The high number of actors involved and the lack of a comprehensive and longterm planning vision have led to the realization of inadequate and incoherent accomplishments. In this sense, ICT can provide the proper tools for managing the necessary inclusive design processes.

A second fundamental matter, related to cultural heritage, is the coexistence of inclusion and conservation needs, which are frequently considered in contrast, although A. Bellini, among the first that have talked about architectural barriers in restoration, remembered "We have many difficulties, insurmountable difficulties, in imaging a monument that has not been produced for men, which is protected and preserved in itself, as an abstraction, and not for fruition. [...]. It is not an asset if it is not usable, pure contemplation does not belong to architecture" (Bellini, 1998). In those circumstances where the goal of accessibility can be in conflict with the needs of conservation, new technologies can represent instruments able to access a particular cultural heritage site and, more in general, to have a better experience of it.

\section{ICT AND INCLUSION: CHALLENGES AND OPPORTUNITIES}

Set the role that ICT can perform in inclusive development processes, it is necessary to understand to whom they are addressed. Although here it is simplified to provide a faster understanding of the topic, the main purpose is to outline a cognitive framework about human diversity. This activity will allow to identify the issues that must be faced when dealing with inclusion and, additionally, the technical features that ICT solutions must possess, in order to guarantee their use by as many people as possible.

\subsection{Inclusive design: answer to real users and real needs}

Traditionally, diversity was always considered an intrinsic characteristic of the person. However, new reflections led to perceive diversity not as an objective feature, but as a related quality, that shows in the interaction between a person and, e.g., the built environment, an object or another person. This arises a new concept of well-being, condition that has been interpreted as what people can be or do in relation to the available resources and their potential capabilities to promote or achieve functioning they value (Sen, 1993).

What has just been said makes essential to develop the necessary knowledge around real human needs, above all for those with special ones. Additionally, the focus on the necessities rather than people's characteristics allows the transition from dedicated design solutions, aimed to solve requirements for specific disabilities, to a fully inclusive view of the project.

For design purposes, it is possible to identify two main categories, able to contain within them the complexity and diversity of human needs:

- Physical needs framework: the necessities that a person presents in moving and using the objects or other elements located in the built environment, regardless of his physical characteristics;

- Sensorial and cognitive needs framework: the necessities that a person presents in relation to the activities of orientation and localization, in addition to the ability to communicate and relate to persons and objects in the space, regardless of his sensorial and cognitive abilities.

From the point of view of the design practice, first, the physical needs framework takes into account the size of the spaces, so they can allow complete free movements despite the use of technical aids. Subsequently, it considers the continuity of the paths, in order that no element prevent the mobility progression, the materials and their state of preservation, which must not be a source of fatigue or danger, and finally the design of the furnishings, so they do not constitute an element of obstacle and are usable by the largest number of people. 
For the sensorial and cognitive needs framework, the main contribution could be the design of explicit, easily perceivable and legible, and implicit, with integrated environmental markers, signage system. In particular, it is important to provide different perceptive channels of communication, to facilitate the use of the residual sensory capabilities of people with disabilities, taking care, however, to avoid overcrowding information, which paradoxically would produce negative effects in the wayfinding process.

\subsection{ICT accessibility features: potentialities and criticalities}

The previous reflections have introduced a first general overview on the issues addressed by the target of inclusion and to which ICT can provide their own contribution. Since this topic will be later deepened in the paper with the proposal of an operative tool, now it seems appropriate to rapidly investigate some potentialities and criticalities that must be taken into account when designing the features of this technology.

With reference to the physical needs framework, if ICT is the only tool through which is possible to experience certain contents, since other physical conditions could prevent access to places and services, it is immediately clear that the main matter is related to the hardware. The latter have to allow everyone to be able to access ICT, regardless of its capabilities and limiting the person's physical effort. Additionally, this requires to consider (assistive) device and software costs so that economic inequalities are not a further element of exclusion (ITU, 2013).

Nevertheless, the main contribution is in relation to the sensorial and cognitive needs framework. Particularly, ICT provides alternative communication channels that can be adapted to different requirements.

In the case of sensorial disabilities, the main limitations are related to those elements in the built environment that convey information, supporting mobility and activities, only through a single sensory channel. Nowadays, ICT provides multiple instruments able to "translate" data through different communication methods. The most common example is the vocal synthesis of written texts, use by people with visual impairments, however, to better understand the potentialities of ICT, it is interesting to introduce a couple examples of services designed for people with hearing impairments.

The first one is VEASYT Live! (Figure 1) an Italian professional interpreting remote service that can be easily accessed through a computer or tablet that allows to have a professional interpreter, available by videoconference, to translate in sign language the communications in different activities and situations.

The second one is recently presented by Google and they are two applications: Live Transcribe is an app for real-time transcription of the voice of the person who is speaking; while, Sound Amplifier will allow to filter, increase and amplify the sounds of the environment in which you are (Figure 2). From their description, it clearly emerges how ICT allows to solve a daily issue, the one of communication, in a more effective and faster way, compared to more "traditional" approaches, limiting costs and the necessity of specific solutions.

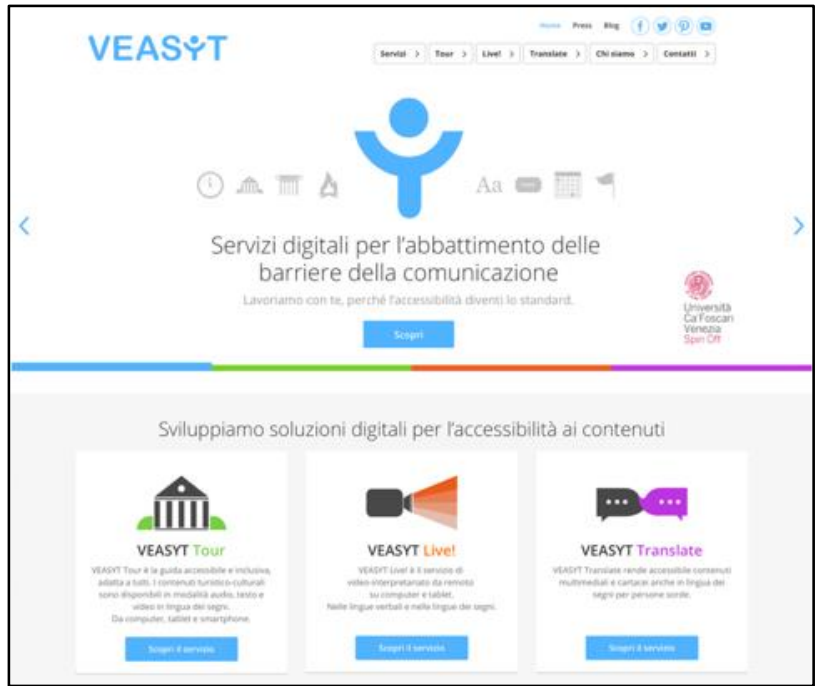

Figure 1. Screenshot of VEASYT Website. Source: http://www.veasyt.com

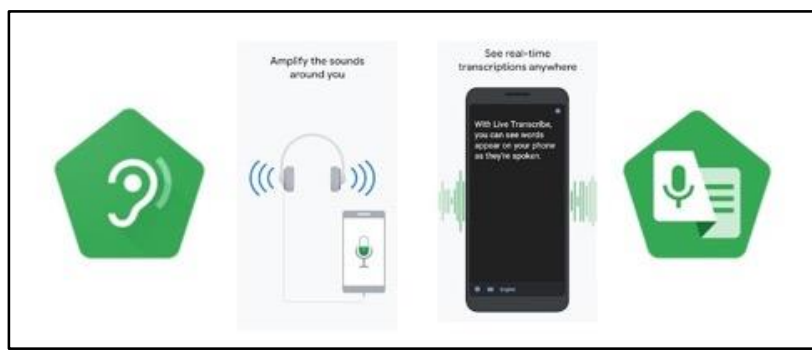

Figure 2. Google's Sound amplifier and Live Transcribe apps Source: https://www.disabili.com/prodotti/articoliqprodotti/sound-amplifier-e-live-transcribe-le-novita-da-googleper-utenti-sordi

The latter aspect also assumes particular significance in relation to cognitive impairments, meant not only referred to disabilities but also to situations like different cultural background, language, etc. Indeed, ICT not only can provide the same information adapted to the different cognitive abilities of the users who requests access from time to time, but it allows to supply the servicer through a single (software and/or hardware) support.

Therefore, to express the described potentialities, the same ICT must be designed to provide the proper answers to different needs, that have to be acknowledged and taken into account, otherwise, as shown by the current condition, single services will never be able to satisfy the wide request of support in accessing information and communication.

\section{INTERACTIVE MAP}

These first analyses allowed us to understand the potentialities that ICT has in favoring inclusion, especially if assessed in relation to the objectives of the enhancement and conservation of cultural heritage sites. For the purposes of the work, the conception of an interactive map has seemed the proper solution in the perspective of producing a feasible operative tool.

At this stage of the research, where the focus is principally in defining the main objectives and features of the map rather than its software architecture, this instrument has seemed the ideal interface to be used. It's easy to think of how, even in the daily travel activities, we resort to the use of digital maps; not only they let us to organize our movements, but they are also one of 
the first tools through which we become aware of a place. In this sense, when the reference is to cultural heritage sites, digitals maps have a key role in collecting and sharing knowledge.

In addition to that, working through a cartographic base, this type of implement allows an easier integration with the existing documents and resources of urban planning, enabling to outline all the initiatives, whether they are architectural projects or not, necessary to allow inclusion to lead the transformation processes of the city.

\subsection{Accessibility and interactive maps: background}

In recent years, the success of digital maps has been widely recognized. This type of services, such as Google Maps, Bing Maps, Yelp and Waze, has contributed to the spread of an increasing number of information, although the data regarding the physical accessibility of the built environment are extremely poor.

For this reason, various initiatives focused on the issue of accessibility have been developed in parallel to these tools. One of the best-known examples is Wheelmap.org (Figure 3), an online map that collects accessibility information that has been reported by the users of its smartphone application, through onsite visits of the different points of interest within a city.

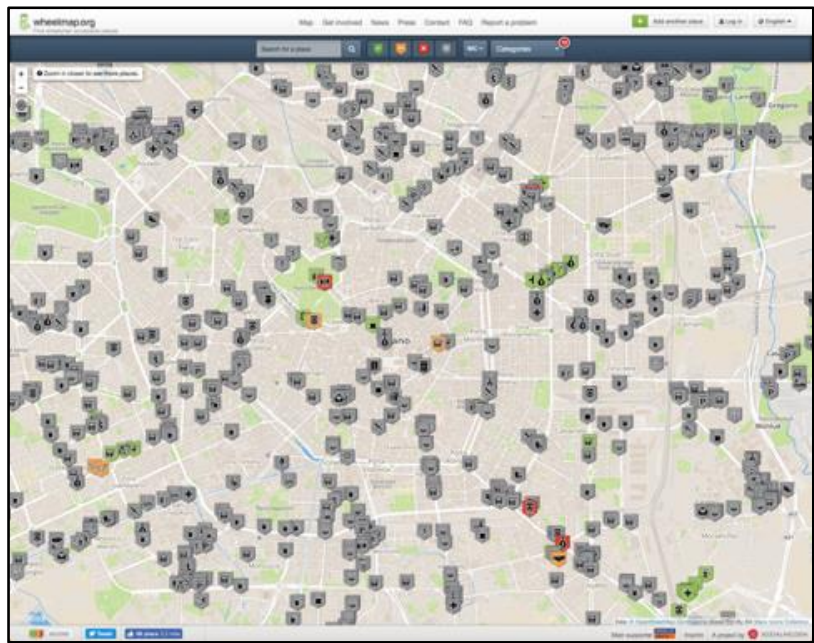

Figure 3. Screenshot of Wheelmap website. In the case of Milan (Italy), it's possible to see how many POIs still have missing information (in grey). Source: http://wheelmap.org

However, this kind of solutions still has several limitations. First of all, they suffer from a lack of records. If we consider the example of Wheelmap.org, a report on open accessibility data unearthed that only the $1,6 \%$ of the points of interest on the map had accessibility data (Ding et al., 2014). In addition, this information refers only to punctual situations, measuring the accessibility of the single facilities without considering the paths that connect them. On the contrary, there are also some services that focus their attention only on the accessibility of the urban environment, like Project Sidewalk (Figure 4). Developed in Washington D.C., this project has been aimed at mapping all the accessibility issues that the city sidewalks present, through virtual exploration of the streets.

Certainly, the data collected establish an important basis to develop inclusive policies, but the absence of information about facilities and other important "urban components", such as public transport, doesn't allow to have a complete vision of the city system and, in the case of end users, to be able to organize your movements. Moreover, these multiple tools also have the side effect of scattering information, since each service contains different one, making it complex for the final user to acquire thorough data about the accessibility of a place.

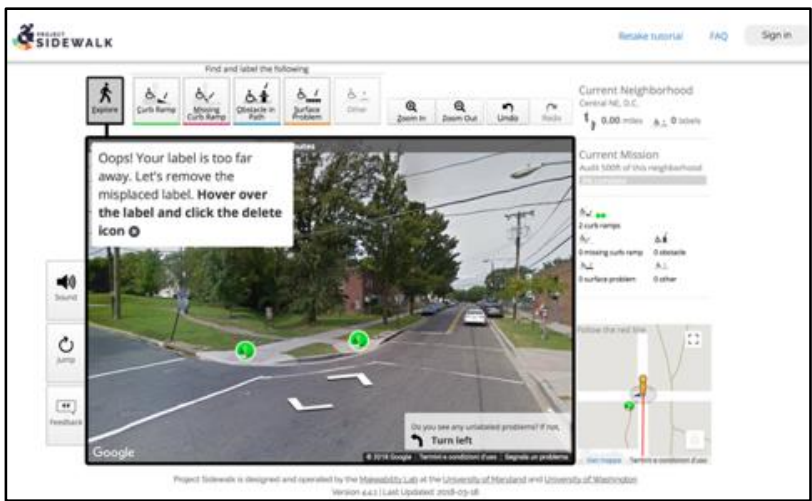

Figure 4. Screenshot of Project Sidewalk website. In this image is visible the user interface through which is possible to map the accessibility issues of the city sidewalks. Source: http://sidewalk.umiacs.umd.edu

A further fundamental aspect that must be taken into consideration is that this kind of instruments are mainly based on crowdsourcing. This model surely allows a faster and more widespread data collection; however, the accuracy of the information can be strongly variable. On the one hand, it can be necessary to evaluate the survey methodologies and their effectiveness, as the experimentation developed by Salomoni $e t$ al. on three different mobile apps to crowdsourcing urban accessibility has shown (Salomoni et al., 2015). On the other hand, it's very important to underline how the concept of accessibility can be interpreted differently from map to map and especially from person to person. In case the data have not been previously verified or no objective indications on the parameters used to define the level of accessibility of a place have been provided, the risk is to produce a strong discrepancy between users' expectation and their actual travel experience, aspect that has been recognized as one of the greatest difficulties disabled people have in traveling (Packer, 2007).

In addition to the previous limitations, it's necessary to draw attention on how these tools are mostly addressed to people with physical disabilities, without considering those affected by sensory or cognitive impairments. In this case, the reference is not only to this kind of parameters, completely missing when defining the level of accessibility of a place. In fact, the fundamental issue is to identify the proper technologies and solutions that allow visually and cognitive impaired users to obtain the same information provided by the map.

\subsection{Interactive maps: objectives}

On the basis of the presented limitations of the existing services and the unanswered needs identified through the case studies and on-site investigation, the following sections of the paper try to contribute to the discussions about the objectives and features that this kind of tools should fulfill. In particular, this interactive map has been conceived to perform two fundamental functions. In accordance with the analysis conducted on existing tools, the primary purpose is to provide an information system for people. Especially for contexts of high tourist attraction, it has emerged how information is indispensable to ensure the ability to access and move in the built environment. In particular, people with disability need to receive as much data as possible, in order to know the level of accessibility of a place. The goal of defining a new interactive map is to offer only one and easy to use tool 
that makes everyone able to organize their movements, according to their personal needs and the possibilities offered to them through the proposed services and fulfilled projects from local institutions.

Furthermore, if properly designed, this map could become the appropriate tool with which citizens can participate in the process of fostering inclusion. In line with the model of crowdsourcing, users can use it to advise the administrative bodies of the critical situations located within the city. Although, for what have been said above, it is necessary a professional supervision, it is important to maintain this element because in this way, not only the sense of social participation can be fostered, but the planned interventions would be aimed at answering to real needs expressed by people, who could guide the design process, ensuring the effectiveness of the design actions.

In relation to what has just been said, there is another important function for which this tool has been considered suitable: the management of the projects related to the development of inclusion, therefore it is addressed to municipal administrators and other key actors, such as public or private institutions involved in the governance of cultural heritage. In this sense, the map should locate within the city all the situations representing a physical barrier that restricts people's public participation. This complete cognitive framework, which should be integrated with recommendations on how to overcome physical barriers, will be able to support the planning of the necessary interventions, in accordance with several parameters, such as priority and financial resources, which can be evaluated from time to time. This kind of tool should guarantee a general and long-term vision, capable of adapting to various external factor that could influence the fulfillment of the initiatives to be undertaken.

Another essential aspect is the possibility of coordination among the different actors involved in the development of inclusion. As it has been said, the latter is defined by multiple components, whose supervision is entrusted to many executives. For this reason, the interactive map should be aimed at be the only tool that everyone can interface with, so the planned actions can be combined and properly realized to reach the highest possible level of inclusion. In addition, if conceived as a single instrument, this managing system can offer the adequate supervision to data coming from crowdsourcing, ensuring the accuracy of the information provided. Finally, this tool should be conceived to allow the continuous monitoring of the achieved inclusion. In fact, after the first surveys, the map should be constantly updated, notifying the issues that have been resolved and the new criticalities that could emerge, always providing the most recent vision of the level of inclusion reached within the city.

\subsection{Interactive maps: features}

Once the two main aims have been defined, the focus of the discussion must necessary move on the features and functions the interactive map should possess.

First of all, this tool should be able to provide information on the presence of architectural barriers within the city, both at urban and building scale, if of public interest. As already mentioned above, this data could be the results of professional surveys led by local institutions or reports from crowdsourcing. For this reason, prior to the development of the map, it is necessary to designate a series of unambiguous parameters that can then be made available on a designated area of the website and smartphone application. This would allow both the records from crowdsourcing to be as objective as possible and the end users to be aware of how the level of accessibility has been defined. In addition, these data should be pointed out in different ways, depending on the identified type of criticality. As it has emerged through the analysis of different experiences and the on-site investigation of the case study of Mantua, it is possible to classify three sorts of physical barriers: the punctual situations (Figure 5), the ones with a linear profile (Figure 6) and those that develop on an extended surface (Figure 7).

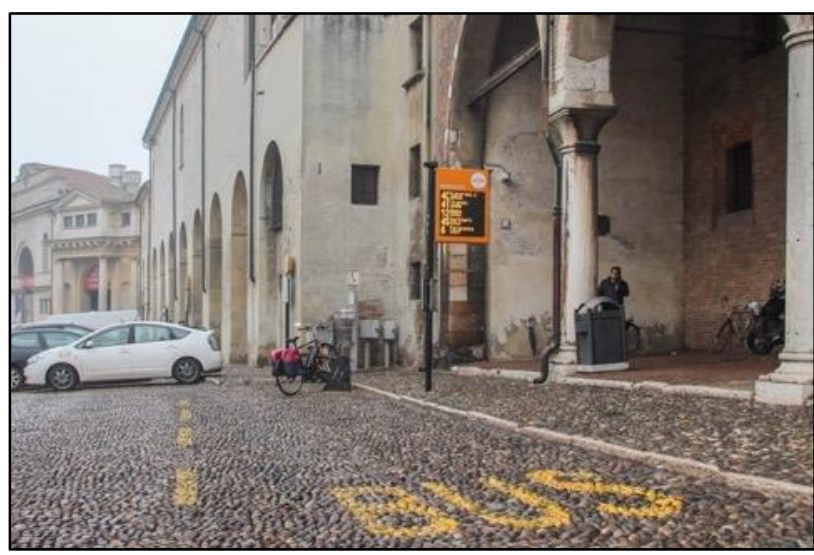

Figure 5. Example of punctual physical barrier: the bus stop in Sordello Square whose characteristics limit its use by people with disabilities. (C) Sebastiano Marconcini, 2017

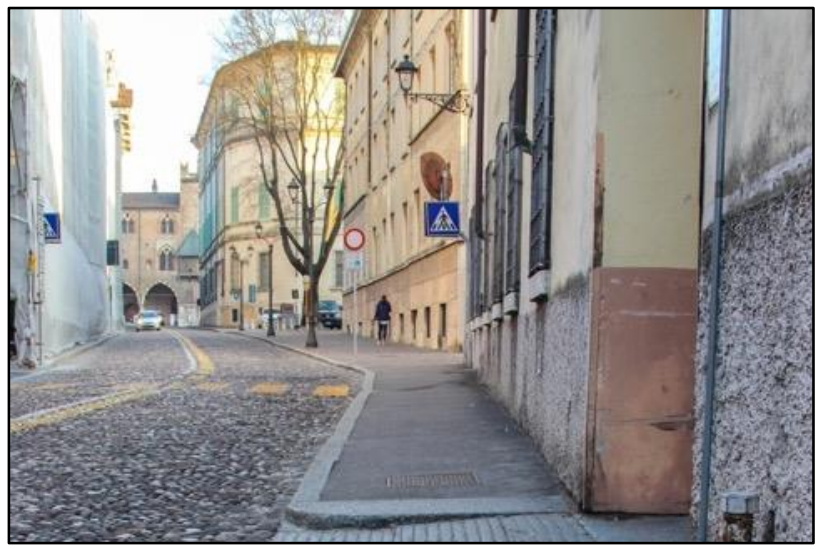

Figure 6. Example of linear physical barrier: sidewalk with excessive transversal, connecting Virgilian Square to Sordello

Square, is a source of fatigue and danger for someone with physical impairments () Sebastiano Marconcini, 2017

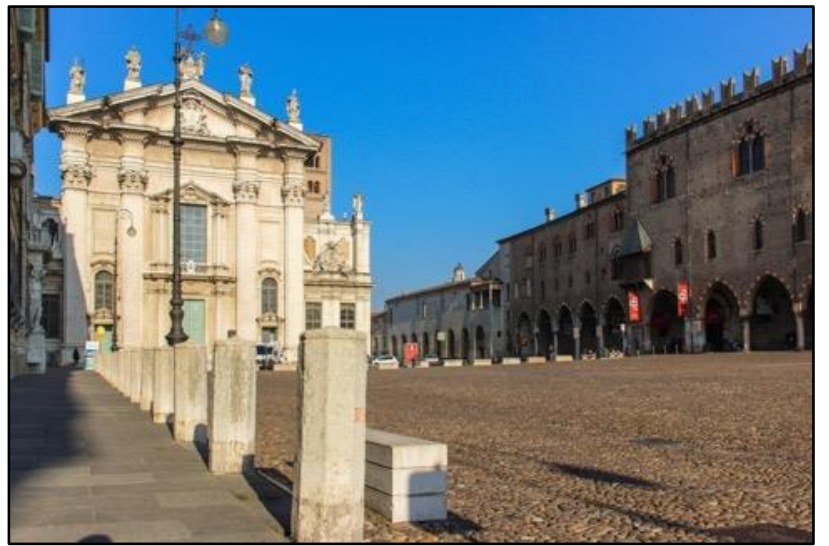

Figure 7. Example of an extended physical barrier: the materials and the excessive slopes of Sordello Square area a source of great inconvenience and danger for people with disabilities (C) Sebastiano Marconcini, 2017 
This distinction is critical both for end users to easily understand the extent of the limitations present and for the management purposes, because it already allows to express some preliminary evaluations on the interventions needed, the difficulty level of overcoming the architectural barriers and the relative costs to do so. The need to have this multiple information requires the map to be structured to provide an overall view of the city and, at the same time, more exhaustive and always updated data related to specific situations. In this sense, the proposed tool should overcome the approach of offering the assertion of the accessibility status, in favor of a more detailed information system that allows the end users to assess, according to their needs, the level of accessibility of a place. For this reason, in order to get this complete cognitive framework, a pop-up window must be associated with every situation identified. In this sense, also multimedia (like images, videos, etc.) should be integrated as an important objective resource for people to evaluate the existing possibilities and organize their movements.

Clearly, if it is essential to identify all the critical situations within the city, the final information that the map needs to offer is different in relation to the two main goals previously define for this tool. From the managing point of view, the key aspects are: the definition of the design proposals, the subdivision of the competencies of the single interventions, so, if necessary, it would be possible to coordinate different actors among themselves, and planning the projects that will be implemented. Instead, as an information system for people, the data the map should supply are of different nature and more general interest. In fact, they should allow a person to identify the points of interest and the paths for reaching them (Figure 8).

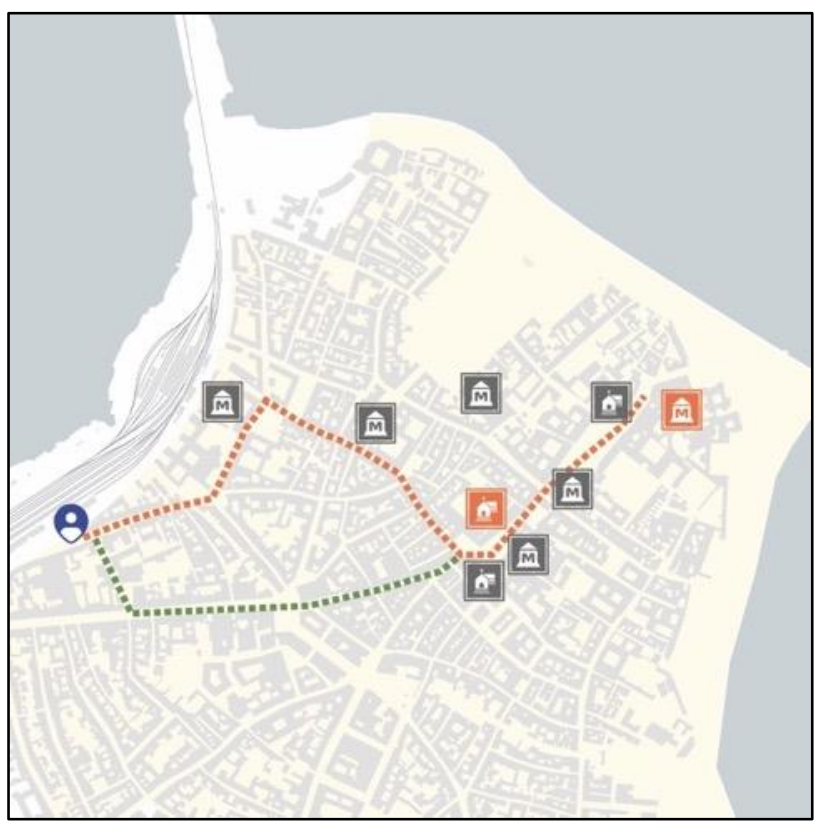

Figure 8. Graphic simulation of the Interactive map proposal for

Mantua. Once selected your POIs, with a general indication about their accessibility given by the colour of the icon, the map provides you the shortest/quickest paths through which reaching them and the nearest POIs related to your research. After a first general overview about the level of their accessibility, it is possible to click on the paths and get deeper information about architectural barriers. (C) Sebastiano Marconcini, 2018
An interesting example that could express the potential of the presented proposal is the app Spot Viborg (Figure 9), developed by the City of Viborg, in Denmark.
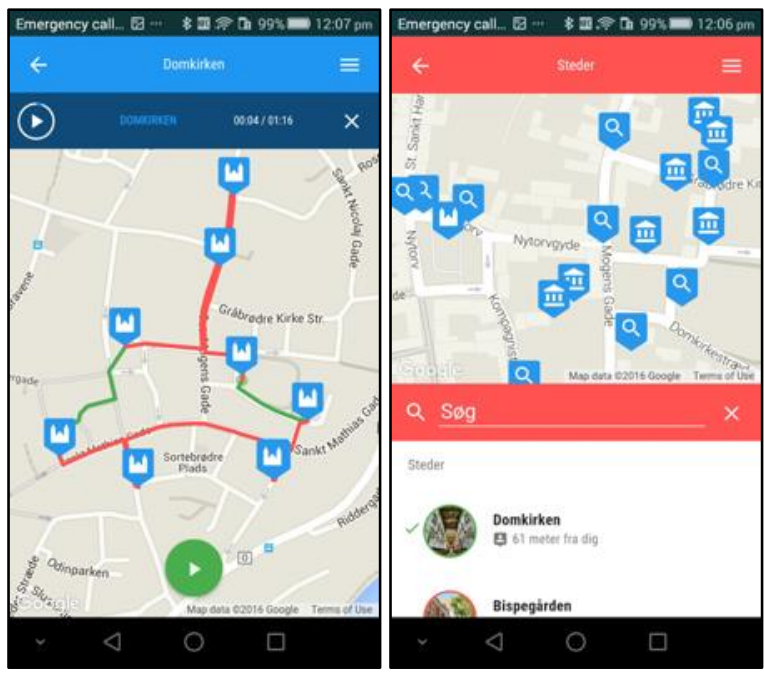

Figure 9. Screenshots of Spot Viborg app. Source:

https://play.google.com/store/apps/details?id=dk.viborgkommu ne.SpotViborg\&hl=it

This application "guides visitors through three itineraries in the historic city. Using GPS tracking, sound and vision, the app also includes information about accessible toilet facilities and parking places. For those who do not want to follow a set itinerary, the app also pinpoints individual attractions leaving the visitor free to create their own route" (European Commission, 2018).

In particular, the tool should be accessed through a user profile, previously set according to the capabilities and consequent needs of the person (Figure 10 and 11). Therefore, this solution would allow to filter all the available data, in order to guarantee the best solutions to move within a place in complete safety and accordance with personal urgencies. In addition, this information should be provided with a simplified language, so everyone can be able to understand them regardless of their cognitive abilities.

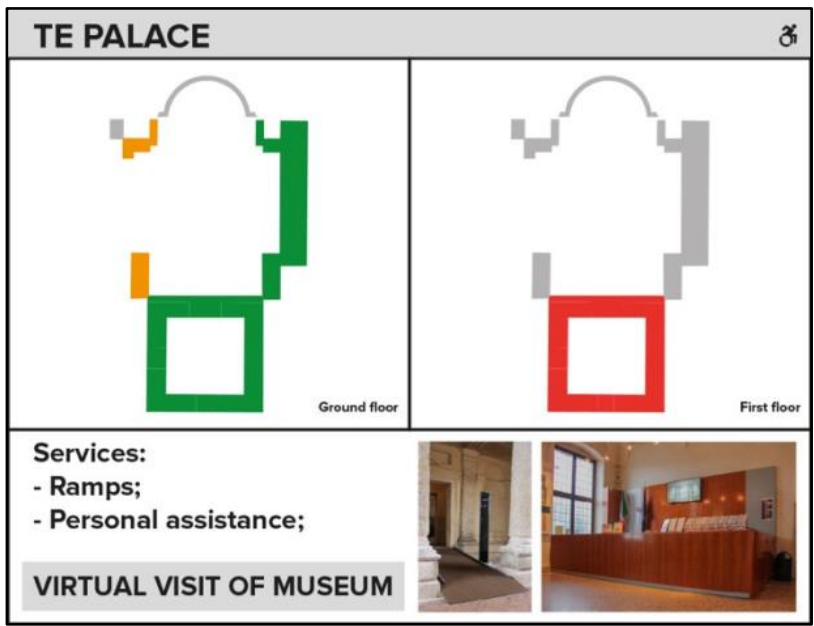

Figure 10. Graphic simulation of a possible pop-up window reachable from the map, after setting the physical impairments user profile, that could provide accessibility information about a specific POIs @ Sebastiano Marconcini, 2018 


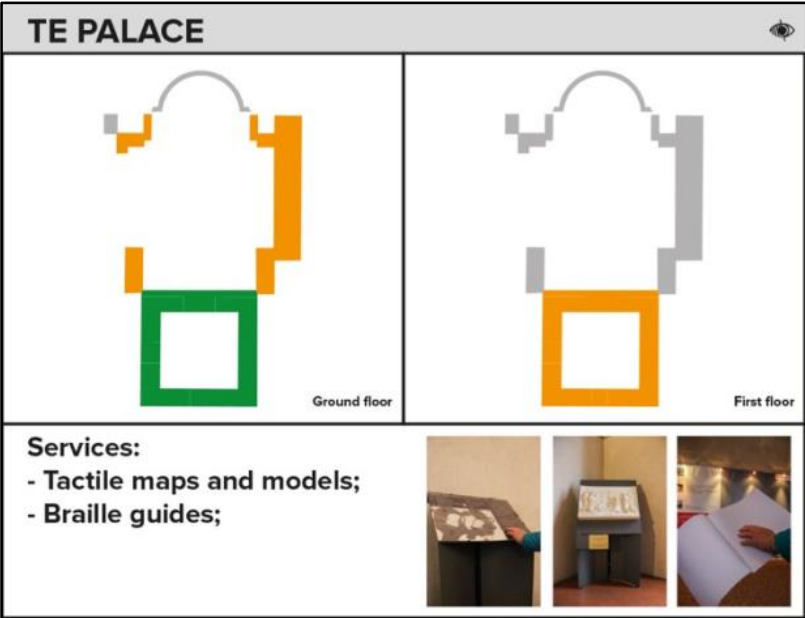

Figure 11. Graphic simulation of a possible pop-up window reachable from the map, after setting the visual impairments user profile, that could provide accessibility information about a specific POIs (C) Sebastiano Marconcini, 2018

Finally, in relation to physical accessibility issues, the information given not only has to focus on architectural barriers, but especially on those elements and services of the built environment, such as public transport, that could enable a better experience of social participation.

\subsection{Beyond interactive maps: ICT services integration}

The proposal of the interactive map has tried to answer to those essential needs that could foster access to cultural heritage sites and inclusive design processes.

Furthermore, this tool could be the user interface through which easily access additional ICT services, that in recent years have undergone or are experiencing a significant technological advancement. Particularly, in addition to the fulfilment of accessibility requirements, these solutions can contribute in the process of sharing knowledge providing a richer experience for everyone, thus fostering social participation and communities' involvement in heritage conservation.

Even without specific competences in this research field, it is possible to highlight how different technologies could be a fundamental integration to achieve the objectives of the interactive map.

One of the main references is related to Virtual Reality and Augmented Reality. In some cases, e.g. where it is not possible to design physical accessibility without compromising the cultural, historical and artistic values of a place, VR can be the only solutions to access/understand cultural heritage, although this should not be a priori alternative. AR represents a remarkable opportunity to provide information, even additional one, without conveying a message through specific linguistic interpretations and adapting to the different users' cognitive abilities.

Despite the complexity of the topic and a wide debate on its opportunities and challenges, it seems proper to mention the Internet of Things (IoT) perspective as an additional resource for the information offered by the interactive map. The arrangement of sensors, iBeacon or human based reporting system would be able to provide updated information in real time about the efficiency of the services of interest (e.g public transport, elevators...), allowing an increasingly precise organization of people's movements in the built environment, especially for those with disabilities.

Finally, a conclusive consideration is needed on GIS and HBIM systems. As previously stated, in order to help in managing the inclusive design processes, the interactive map has been designed to combine with other planning tools and fully integrate the topic of inclusion into sustainable development strategies. For how they are conceived, these technologies perfectly fit for this purpose, since they can coordinate the initiatives and the actors involved in the processes of urban transformation or building conservation, optimizing planning in relation to different parameters, such as priorities, economic resources and many more. Accordingly, the research field must provide a further integration of these technologies to foster their unexpressed potentialities in inclusion development.

\section{CONCLUSION}

Through the discussions about the main objectives and features to which an interactive map should respond, it has been possible to highlight the new role that ICT can assume within an inclusive design approach. For the development of a fully inclusive environment, it is no longer enough to work on the component of physical accessibility, but also on the ones, like information, belonging to a managing dimension.

The proposal of a new operative tool made it possible to transfer, in the ICT sector, the same measures that an inclusive design approach should implement, analyzing limitations and perspectives of these instruments.

As a conclusion it must be underlined that this topic is nowadays considered crucial and supported by National and European Community policies who consider the participative access to cultural policies able to have social, economic and health positive consequences.

Up to now, the elderly or disabled population were often represented only in terms of unexpressed economic potential, as shown for example by studies dealing with cultural tourism. Accessible tourism, the "barrier-free" tourism, is a force worth 3\% of the European Union's GDP: in 2012 it generated revenues of 786 billion and 9 million jobs. However, only 9\% of tourist sites and services in Europe are accessible. There is therefore a huge question that represents an opportunity that it is possible to intercept.

Also from a social point of view, to define the possible benefit, it must be underlined that access and participation are closely terms. Related policies aim to ensure equal opportunities of enjoyment of culture through the identification of underrepresented groups, the design and implementation of initiatives or programs aimed at increasing their participation, and the removal of barriers.

In short, we consider the enhancement of the accessibility' levels of Cultural Heritage as a collective resource, involving social, civil, ethic and environmental aspects, all of them parts of an open and extended concept of sustainable economy. Accessibility can thus become the amount of civilization that our era delivers to the future and can help to improve social cohesion, thereby becoming an act of democracy.

\section{REFERENCES}

Bellini, A., 1998. La pura contemplazione non appartiene all'architettura, Tema, 1, 2-3

CHCfE Consortium, 2015. Cultural Heritage Counts for Europe, full report

Council of Europe, 2005. Convention on the Value of Cultural Heritage for Society (Faro Convention), Available at: https://www.coe.int/en/web/conventions/full-list/- 
Ding C., Wald M. and Wills G., 2014. A survey of Open Accessibility Data, Web for All Conference

Disabili.com [online], Sound amplifier and Live Transcribe:Le novità da Google per utenti sordi, Available at: https://www.disabili.com/prodotti/articoli-qprodotti/soundamplifier-e-live-transcribe-le-novita-da-google-per-utenti-sordi [Accessed February, 28th 2019]

European Commission, 2018. Access City Award 2018: Examples of best practice for making EU cities more accessible, Luxembourg: Publications Office of the European Union

ITU, 2013. The ICT opportunity for persons with disabilities, in The ICT Opportunity for a Disability-Inclusive Development Framework, ITU, Geneva

Licciardi G., Amirtahmasebi R. (2012), The economics of uniqueness. Investing in historic city cores and cultural heritage assets for sustainable development, The World Bank: Washington

Maslow, A.H., 1943. A theory of human motivation, Psychological review, 50(4), 370

Packer T. L., McKercher B., Yau M. K., 2007. Understanding the complex interplay between tourism, disability and environmental contexts, Disability and rehabilitation 294281 292

Project Sidewalk [online] Available at:

http://sidewalk.umiacs.umd.edu [Accessed January, 8th 2019]

Salomoni P., Prandi C., Roccetti M., Nisi V. and Nunes N. J., 2015. Crowdsourcing urban accessibility: Some preliminary experiences with results, Proceedings of the 11th Biannual Conference on Italian SIGCHI Chapter 130-133

Sen, A., 1993. Capability and Well-being. In Sen, A. \& Nussbaum, M. (ed.), The Quality of Life, Claredon Press, Oxford

United Nations, 2006. UN Convention on the Rights of Persons with Disabilities (United nations)

VEASYT Live! [online] Available at: http://www.veasyt.com [Accessed January, 8th 2019]

Wheelmap.org [online] Available at: http://wheelmap.org [Accessed January, 8th 2019]

World Health Organization, 2001. International Classification of Functioning, Disability and Health: ICF, World Health Organization 